\title{
Alzheimer's disease: the potential therapeutic role of the natural antibiotic amyloid- $\beta$ peptide
}
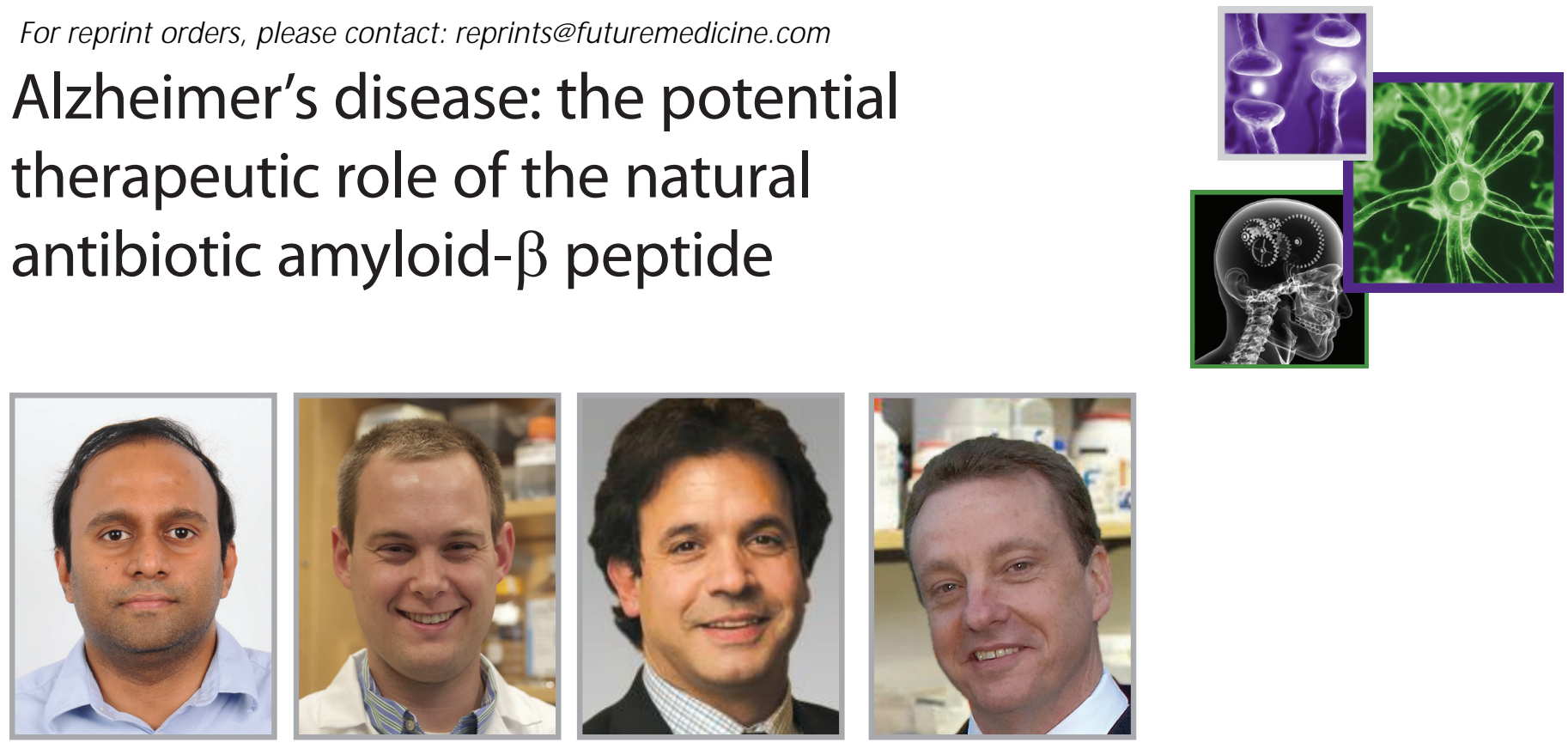

Deepak Kumar Vijaya Kumar', William A Eimer'1, Rudolph E Tanzi' \& Robert D Moir ${ }^{*}$

First draft submitted: 1 August 2016; Accepted for publication: 11 August 2016; Published online: 7 September 2016

Innate immunity is the oldest arm of host defense. Among invertebrates, innate immunity remains the primary defense against infection. Higher organisms have evolved additional adaptive immune responses but the pathways of innate immunity continue to play essential protective roles, including early recognition of infection in tissues, direct inhibition of pathogens through release of antimicrobial molecules, phagocytic removal of cellular debris and foreign materials by leukocytes, recruitment of immune cells to sites of infection through the release of cytokines, alerting adaptive immunity to potential threats through the complement cascade and antigen presentation, and providing chemical and physical barriers to infectious agents [1]. While innate immune responses are rapid and provide immediate host protection they are generic in that the same protective pathways are activated with widely differing immune challenges. The primary effector molecules of innate immunity are antimicrobial peptides (AMPs), also called host defense peptides. AMPs are small (typically $<50$ amino acids) amphipathic peptides and are expressed by both single and multicellular organisms [2]. Microbicidal and microbiostatic pathways are the most conspicuous and widely studied AMP activities. However, vertebrate AMPs are highly pleotropic in their actions and mediate a broad range of innate immune responses. The immunomodulatory activities of AMPs appear to be at least as important for host protection as the peptides direct antimicrobial actions. While essential for normal immunocompetence, mounting evidence suggests that AMPs also have a dark side. Overexpression or dysregulation of AMP pathways are implicated in the etiology of a range of chronic human diseases [3].

We recently demonstrated that the amyloid- $\beta$ peptide $(A \beta)$ of Alzheimer's disease (AD) is a potent AMP and protects against infections in vivo $[4,5] . A \beta$ is an ancient and highly conserved molecule in

\section{KEYWORDS}

- Alzheimer's disease $\bullet$ amyloid- $\beta$

- infection

'Genetics \& Aging Research Unit, Department of Neurology, Massachusetts General Hospital \& Harvard Medical School, Charlestown, MA 02129, USA

*Author for correspondence: Tel.: +1 617726 3746; moir@helix.mgh.harvard.edu

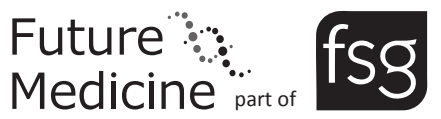




\author{
"Such oligomer \\ preparations are likely \\ to more closely model the \\ highly efficient synergistic \\ actions of endogenous \\ antimicrobial peptides \\ in vivo."
}

over $70 \%$ of vertebrate species [6]. The remarkable conservation across vertebrates and high antimicrobial activity in animal models [5] suggest $A \beta$ is an important and potent natural AMP. This has important implications for the peptide's pathological actions in AD. However, an AMP function for $A \beta$ also raises new and intriguing possibilities independent of the peptide's pathological role. Namely, could $A \beta$ or $A \beta$ derivatives be developed into clinically useful agents? The last decade has seen considerable effort to develop natural and synthetic AMPs as clinical agents to treat disease. Efforts have mostly focused on developing AMPs as peptide antibiotics. Over 20 clinical trials have been conducted using AMPs as either topical or systemic antibiotics [7]. $A \beta$ or $A \beta$ derivatives may have potential as clinical antibiotics. In addition, we believe there may also be opportunities for advancing peptide-based therapies by using $A \beta$ as a model of AMP actions in vivo, particularly those protective activities mediated by peptide oligomerization.

Oligomerization mediates at least two separate but overlapping $A \beta$ antimicrobial pathways. The first is a classical AMP pathway and involves perturbation of microbial membranes by oligomeric $A \beta$ species. Soluble $A \beta$ oligomers bind microbial surface, then fibrilize disrupting membrane integrity. Membrane disruption pathways are the most widely characterized AMP microbicidal mechanism and the focus of most efforts to develop peptide antibiotics. However, microbial membrane disruption typically requires micromolar AMP concentrations. $A \beta$, and a majority of classical AMPs, are normally expressed at low nanomolar concentrations. At low nanomolar levels, a second $A \beta$ pathway involving microbe agglutination and sequestration inhibits invading pathogens. In this pathway, soluble $A \beta$ oligomers target and bind microbial surface carbohydrates. Oligomeric $A \beta$ species possess enhanced carbohydrate-binding properties and are key for microbe targeting. Microbial cell wall binding by soluble $A \beta$ oligomers is increased three to four orders of magnitude $(\log 10)$ compared with monomeric peptide [8]. Once bound, oligomers provide a nidus and anchor for $A \beta$ fibril propagation. Growing $A \beta$ fibrils capture, agglutinate and finally entrap microbes in a network of $\beta$-amyloid. Agglutination generates high local $A \beta$ concentrations within agglutinates and facilitates microbial killing by membrane perturbation pathways. $A \beta$ oligomerization is key for the peptides targeting and inhibition of pathogens. Available data suggest that classical AMPs also generate soluble oligomeric species that mediate microbial targeting and inhibition pathways. However, therapeutic strategies aimed at developing clinically useful peptide antibiotics rarely consider the potential benefits of soluble oligomeric AMP species. Opsonization is another immune pathway with potential for increasing AMP clinical efficacy. In opsonization, surface-bound AMP oligomers mark a microbe for phagocytosis [9]. Administering combinations of specific pre-assembled oligomers may offer an effective strategy for targeting pathogens with a diverse array of protective AMP activities, including agglutination and opsonization pathways. Such oligomer preparations are likely to more closely model the highly efficient synergistic actions of endogenous AMPs in vivo.

Soluble $A \beta$ oligomers are integral to the antimicrobial activities of $A \beta$. Consistent with a key physiological role for multimer species, the pool of soluble $A \beta$ in brain is overwhelmingly comprised of polydispersed polymorphic oligomers [10]. A $\beta$ oligomers show antimicrobial activities that are broader and two to three orders of magnitude $(\log 10)$ more potent than homodisperse monomeric peptide [5]. In addition to agglutination and opsonization, soluble AMP oligomers have been shown to mediate a range of activities important for host protection, including pathogen targeting [11], microbial cell membranes disruption [12], increase resistance to proteases that inactivate host immune peptides [13] and broadening the spectrum of microbes targeted by AMP families [8]. A large body of data is available on the structure, physiochemistry and activities of $A \beta$ oligomers. It seems likely this data contain useful insights into oligomer-mediated activities of classical AMPs and may also help guide future efforts to develop specific multimer configurations that increase the efficacy of peptide antibiotics.

$\mathrm{A} \beta$ oligomer models may prove especially helpful in overcoming the most often cited challenges to using AMPs as antibiotic drugs [7], including: first, high dose required for efficacy; second, high cost of peptide production and third, high vulnerability to environmental degradation. $A \beta$ oligomers show robustly increased potency, specificity and stability. Enhanced antimicrobial activities for oligomers of classical AMPs would reduce the dosage required for effective antibiotic actions. Lower doses would 
reduce drug costs per patient. The potential to direct or broaden pathogen specificity through generation of specific oligomeric species would also expand possible applications available for single peptide products. This would help consolidate peptide synthesis processes and further reduce production costs. Finally, the high stability of $A \beta$ multimers suggests that AMP oligomer preparations are also likely to resist environmental degradation and show enhanced shelf-lives compared with monomeric peptides. Soluble AMP oligomers may also offer an additional incentive for the pharmacological industry. Soluble oligomer preparations would be categorized as biopharmaceuticals. Biologics generated by a production process of controlled oligomerization are likely to be considerably more difficult and costly to replicate/authenticate than traditional generic drugs, which only duplicate a simple chemical structure.

In addition to providing a model for peptide antibiotic development, there are potential clinical applications for $A \beta$ itself. The in vitro antimicrobial $A \beta$ activities match, and in some cases exceed, those of AMPs previously trialed as antibiotics $[4,5]$. $A \beta$ also shows a broad activity spectrum, showing activity against fungi, bacteria and viruses. Thus, data suggest that $A \beta$ or $A \beta$ derivatives are feasible candidates for development as peptide antibiotics. Potential clinical uses for $A \beta$ also extend beyond use as an antibiotic. LL-37 is an archetypal human AMP that has been our model for the antimicrobial actions of A $\beta$. LL-37 is currently being trialed as an anticancer drug (identifier NCT02225366 at ClinicalTrials.gov). Studies have not focused on the anticancer activities of $A \beta$. However, human $A \beta$ expression in transgenic mice has been reported to inhibit the growth of tumors transplanted into the brain [14]. The cytotoxic mechanism appears specific for glioma cells. AMPs are also potent cytokines and are currently being explored as immunomodulator drugs [15]. $A \beta$ in the CNS is proinflammatory but potently anti-inflammatory when administered in the periphery [16]. However, for over three decades $A \beta$ has been associated with $A D$ pathology and several potential safety concerns will need to be addressed before the peptide can be used as a drug. Most prominent is the potential for $A \beta$ host cytotoxicity. $A \beta$ is frequently characterized as 'highly cytotoxic'. However, among eukaryotic cells, high $A \beta$ cytotoxicity appears limited to neurons. Moreover, neurotoxicity requires highly specific and uncommon $A \beta$ oligomer species. Indeed, generating experimental $A \beta$ species with consistent neurotoxic activity has proved a major challenge [17]. The overwhelming majority of monomeric and oligomeric $A \beta$ species do not appear cytotoxic toward neurons or other eukaryotic cells. Broad cytotoxicity against eukaryotic cells requires high $\mathrm{A} \beta$ concentrations $(>50 \mu \mathrm{M})$ that typically exceed levels likely to be used clinically. Indeed, many classical AMPs show broad host cytotoxicity at considerably lower concentrations than A $\beta$. The adage 'dose makes the poison' remains as relevant today as when first expressed over five centuries ago. At the concentrations likely to be used clinically, $A \beta$ host cytotoxicity seems unlikely to preclude the peptides use as an antibiotic. Moreover, selectively removing or inhibiting the generation of rare problematic species in $A \beta$ preparations seems a viable strategy should neurotoxicity prove a problem.

$\beta$-amyloid deposition in host tissues is also a likely concern for systemic use of an $A \beta$ drug. $A \beta$ is normally expressed in blood and peripheral tissues. However, $A \beta$ outside the brain has not been reported to lead to amyloidosis. $\beta$-amyloid deposited in the periphery may be cleared by the immune system. This model is consistent with findings for the protective actions of $A \beta$ autoantibodies and $A D$ immune therapies that stimulate immune-mediated clearance of $\beta$-amyloid from brain [18]. However, it remains unclear if immune-mediated clearance would be sufficiently robust to clear levels of exogenous $A \beta$ administered as a therapeutic agent. For clinical trials, natural AMPs with high host cytotoxicity have been modified to reduce toxicity [7]. A possible strategy to limit amyloid generation in tissues may be to use modified human $A \beta$ or nonhuman sequences. Rodent and human $A \beta$ sequences diverge by three amino acids. Rodent $A \beta$ generates diffuse deposits with infection that are more readily cleared than human $\beta$-amyloid plaques [19]. Use of rodent $A \beta$ peptides may reduce potential problems associated with systemic $\beta$-amyloid accumulation.

It remains to be determined if $A \beta$ is suitable for use as a clinical antibiotic, anticancer or immunomodulator drug. However, an analysis of available data suggests that serious consideration should be given to exploring possible therapeutic applications for this ancient and highly conserved immune molecule.

In summary, we believe $A \beta$ may be useful for advancing development of peptide antibiotics.

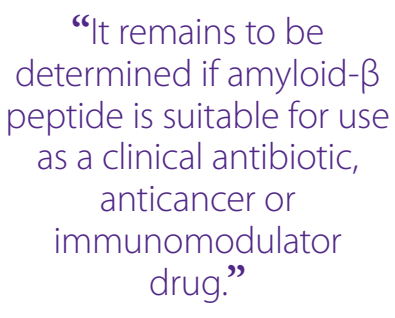


Firstly, $A \beta$ or $A \beta$ derivatives may themselves be candidate therapeutic agents. However, safety concerns will need to be addressed as to the potential for $\beta$-amyloid accumulation in patient peripheral tissues. Secondly, A $\beta$ may provide a useful model for development of soluble AMP oligomers as therapeutic agents. AMP oligomers are likely to show increased potency, specificity and stability compared with the monomeric peptides used in clinical trials to date. Enhanced antimicrobial efficacy for peptide oligomers may have the additional benefit of lowering current high costs of AMP antibiotic therapies. The emerging role of innate immune pathways in AD pathology is helping change how $\beta$-amyloid deposition in brain is viewed. However, the emerging AMP identity for $A \beta$ has also revealed the potential for new beneficial roles for a peptide long perceived only as an intractable enemy.

\section{Financial \& competing interests disclosure}

This work is supported by grants from NIH (5R01A1081990-02), the Cure Alzheimer's Fund and The Helmsley Charitable Trust. The authors have no other relevant affiliations or financial involvement with any organization or entity with a financial interest in or financial conflict with the subject matter or materials discussed in the manuscript apart from those disclosed.

No writing assistance was utilized in the production of this manuscript.

\section{References}

Papers of special note have been highlighted as:

- of interest; $\bullet$ of considerable interest

1 Flajnik MF, Du Pasquier L. Evolution of innate and adaptive immunity: can we draw a line? Trends Immunol. 25(12), 640-644 (2004).

2 Wiesner J, Vilcinskas A. Antimicrobial peptides: the ancient arm of the human immune system. Virulence 1(5), 440-464 (2010).

3 Zaiou M. Multifunctional antimicrobial peptides: therapeutic targets in several human diseases. J. Mol. Med. 85(4), 317-329 (2007).

4 Soscia SJ, Kirby JE, Washicosky KJ et al. The Alzheimer's disease-associated amyloid $\beta$-protein is an antimicrobial peptide. PLoS ONE 5(3), e9505 (2010).

-• First report showing a possible physiological function for amyloid- $\beta$ peptide $(A \beta)$ as an antimicrobial peptide (AMP).

5 Kumar DK, Choi SH, Washicosky KJ et al. Amyloid- $\beta$ peptide protects against microbial infection in mouse and worm models of Alzheimer's disease. Sci. Transl. Med. 8(340), 340ra372 (2016).

-• Confirms that $A \beta$ protects against infection in animal models, and first report of a physiological role for $\beta$-amyloid.

6 Luna S, Cameron DJ, Ethell DW. Amyloid- $\beta$ and APP deficiencies cause severe cerebrovascular defects: important work for an old villain. PLoS ONE 8(9), e75052 (2013).

7 Jenssen H, Hancock REW. Therapeutic potential of HDPs as immunomodulatory agents. Methods Mol. Biol. 618, 329-347 (2010).

8 Lehrer RI. Paradise lost and paradigm found. Nat. Immunol. 5(8), 775-776 (2004).

- Proposes that 'mix and match' AMP oligomers increase molecular diversity and broaden antimicrobial activity spectrum.

9 Shah C, Hari-Dass R, Raynes JG. Serum amyloid $\mathrm{A}$ is an innate immune opsonin for Gram-negative bacteria. Blood 108(5), 1751-1757 (2006).

10 Yang T, Hong S, Malley TO, Sperling RA, Walsh DM, Selkoe DJ. New ELISAs with high specificity for soluble oligomers of amyloid $\beta$-protein detect natural $\mathrm{A} \beta$ oligomers in human brain but not CSF. Alzheimers Dement. 9(2), 99-112 (2013).

11 Sood R, Domanov Y, Pietiäinen M, Kontinen VP, Kinnunen PKJ. Binding of LL-37 to model biomembranes: insight into target vs host cell recognition. Biochim. Biophys. Acta. 1778(4), 983-996 (2008).

- Shows that AMP oligomers mediate important AMP activities.

12 Oren Z, Lerman JC, Gudmundsson GH, Agerberth B, Shai Y. Structure and organization of the human antimicrobial peptide LL-37 in phospholipid membranes: relevance to the molecular basis for its non-cell-selective activity. Biochem. J. 341(Pt 3), 501-513 (1999).

13 Raimondo D, Andreotti G, Saint N et al. A folding-dependent mechanism of antimicrobial peptide resistance to degradation unveiled by solution structure of distinctin. Proc. Natl Acad. Sci. USA 102(18), 6309-6314 (2005).

14 Paris D, Town T, Parker TA et al. Inhibition of Alzheimer's $\beta$-amyloid induced vasoactivity and proinflammatory response in microglia by a cGMP-dependent mechanism. Exp. Neurol. 157(1), 211-221 (1999).

15 Mansour SC, Pena OM, Hancock RE. Host defense peptides: front-line immunomodulators. Trends Immunol. 35(9), 443-450 (2014).

16 Grant JL, Ghosn EEB, Axtell RC et al. Reversal of paralysis and reduced inflammation from peripheral administration of $\beta$-amyloid in $\mathrm{TH} 1$ and $\mathrm{TH} 17$ versions of experimental autoimmune encephalomyelitis. Sci. Transl. Med. 4(145), 145ra105 (2012).

17 Kayed R, Lasagna-Reeves CA. Molecular mechanisms of amyloid oligomers toxicity. J. Alzheimers Dis. 33(Suppl. 1), S67-S78 (2013).

18 Lindhagen-Persson $\mathrm{M}$, Brännström K, Vestling M, Steinitz M, Olofsson A. Amyloid- $\beta$ oligomer specificity mediated by the IgM isotype - implications for a specific protective mechanism exerted by endogenous auto-antibodies. PLoS ONE 5(11), e13928 (2010).

19 Little CS, Hammond CJ, MacIntyre A, Balin BJ, Appelt DM. Chlamydia pneumoniae induces Alzheimer-like amyloid plaques in brains of BALB/c mice. Neurobiol. Aging 25(4), 419-429 (2004).

- Shows that infection can lead to deposition of endogenous murine $A \beta$ in a mouse model. 